\title{
UTILIZAÇÃO DE MATRIZES DE JULGAMENTOS NA ANÁLISE DO CONTROLE DA PRODUÇÃO
}

\section{THE USE OF JUDGMENT MATRICES IN THE PRODUCTION CONTROL ANALYSIS}

\author{
Valério A. P. Salomon ${ }^{1}$; Tamio Shimizu ${ }^{2}$ \\ 1Universidade Estadual Paulista - UNESP - Guaratinguetá - Brasil \\ salomon@,feg.unesp.br \\ ${ }_{2}$ Universidade de São Paulo - USP - São Paulo - Brasil \\ tmshimiz@usp.br
}

\begin{abstract}
Resumo
Este artigo trata da aplicação da tomada de decisão com múltiplos critérios na análise do controle da produção. Apresenta-se a utilização de matrizes de julgamentos para a análise de atributos quantitativos. Devido às características dos atributos e dos valores de desempenho das alternativas, a utilização de um método de tomada de decisão com múltiplos critérios, com matrizes de julgamentos, provê resultados adequados e uma análise concisa do desempenho das alternativas de controle da produção.
\end{abstract}

Palavras-chave: AHP, Controle da Produção, MCDM.

\section{Introdução}

Este artigo trata da aplicação da MCDM (Multiple Criteria Decision Making, ou Tomada de Decisão com Múltiplos Critérios) na análise do controle da produção. O ponto de partida é o estudo de ROBERT W. BRENNAN e WILLIAM O. (2004) sobre situações com distúrbios no ambiente de produção como, por exemplo, o atraso no processamento em uma máquina. O conceito de "controle da produção" é o mesmo adotado por Silver et al. (1998) e Slack et al. (2002). De acordo com Silver et al. (1998), são funções do controle da produção: a aceitação de listas de expedição, a atualização dostatus da produção e de registros de estoques e o feedback dos desvios no plano mestre.

Para um sistema de produção em lotes ou em série, em situações em que ocorrem distúrbios, dois atributos do controle da produção são: o Tempo Médio de Produção e a Freqüência de Reprogramação (CAVALIERI et al., 1999; BRENNAN e O, 2004). Tratam-se de atributos 
quantitativos inversos. Ou seja, quanto maior o valor, pior o desempenho da alternativa. Quando existem dados numéricos para estes atributos, a harmonização dos dados é uma maneira simples para se analisar o desempenho das alternativas nestes atributos. Porém, a harmonização dos dados de Brennan e O (2004), apresentada na Seção 2 deste artigo, não se mostrou adequada. Assim, na Seção 3, um método de MCDM, com matrizes de julgamentos, foi utilizado, obtendo-se resultados adequados e mais concisos que os originais, que apontava para três de quatro alternativas como as de melhor desempenho.

\section{Análise do desempenho de alternativas de controle da produção}

Brennan e O (2004) estudaram um sistema de produção com quatro estações de trabalho, cada uma com duas máquinas do mesmo tipo. O controle da produção era responsável pelo monitoramento da execução do plano de produção, além da detecção e correção de falhas. Uma consideração importante com relação ao controle da produção é a habilidade do sistema reagir a distúrbios no ambiente produtivo, por exemplo, quebra de uma máquina ou falta de matéria-prima. No caso de ocorrência de um distúrbio, quatro alternativas de controle foram estudadas (DIST, NO_WAIT, WAIT e WAIT_INTEL). As alternativas utilizadas no experimento estão resumidas a seguir:

- DIST - ignoram-se as listas de programação e utiliza-se uma regra de seqüenciamento que privilegia pedidos com a maior soma de tempo de processamento de operações não programadas;

- NO_WAIT - o sistema de produção pede, imediatamente, uma reprogramação sempre que um distúrbio atrasa uma máquina;

- WAIT - o sistema de produção apenas espera o distúrbio passar e nunca pede uma reprogramação;

- WAIT_INTEL - quando o distúrbio atrasa uma máquina, o sistema de produção decide se espera ou se pede uma reprogramação, baseado em uma estimativa de data mais tarde de início (isto é, o tempo o mais tarde que uma estação de trabalho pode ser atrasada sem retardar as operações restantes na programação).

Um atributo do controle da produção é o Tempo Médio de Fluxo, obtido por simulação, ora alterando-se a variabilidade do processamento, ora alterando-se a probabilidade de ocorrência de distúrbios. Outro atributo utilizado é a Freqüência de Reprogramação. A Tabela 1 apresenta os 
valores do Tempo Médio de Fluxo (em minutos) obtidos nos experimentos e a Freqüência de Reprogramação.

Tabela 1 - Desempenho das alternativas de controle da produção

\begin{tabular}{|c|c|c|c|}
\hline \multicolumn{2}{|c|}{ Tempo Médio de Fluxo (min) } \\
\hline Alternativa & $\begin{array}{c}\text { \{ Probabilidade de } \\
\text { Distúrbio Constante }\end{array}$ & $\begin{array}{c}\text { \{ Variabilidade do } \\
\text { Processamento } \\
\text { Constante\} }\end{array}$ & $\begin{array}{c}\text { Freqüência de } \\
\text { Reprogramação }\end{array}$ \\
\hline DIST & 139 & 170 & 57 \\
\hline NO_WAIT & 145 & 172 & 0 \\
\hline WAIT & 167 & 183 & 32 \\
\hline
\end{tabular}

Fonte: Brennan e O (2004)

Brennan e O (2004) concluem que "as alternativas que permitem reprogramação da produção para tratar de distúrbios resultam num melhor desempenho". Analisando apenas os valores de Tempo Médio de Fluxo obtido em cada experimento, as alternativas DIST e WAIT_INTEL apresentam valores de desempenho bem próximos. Mas, não se pode afirmar que estas alternativas apresentam desempenho melhor que NO_WAIT. Uma vantagem da WAIT_INTEL é uma redução significativa na Freqüência de Reprogramação.

Para se obter uma conclusão mais concisa, pode-se aplicar um método de MCDM. O método utilizado neste trabalho é o Analytic Hierarchy Process (AHP), apresentado em Saaty (1980). A Figura 1 apresenta a análise do desempenho das alternativas de controle da produção, com o método AHP.

Figura 1 - Análise do desempenho de alternativas de controle da produção

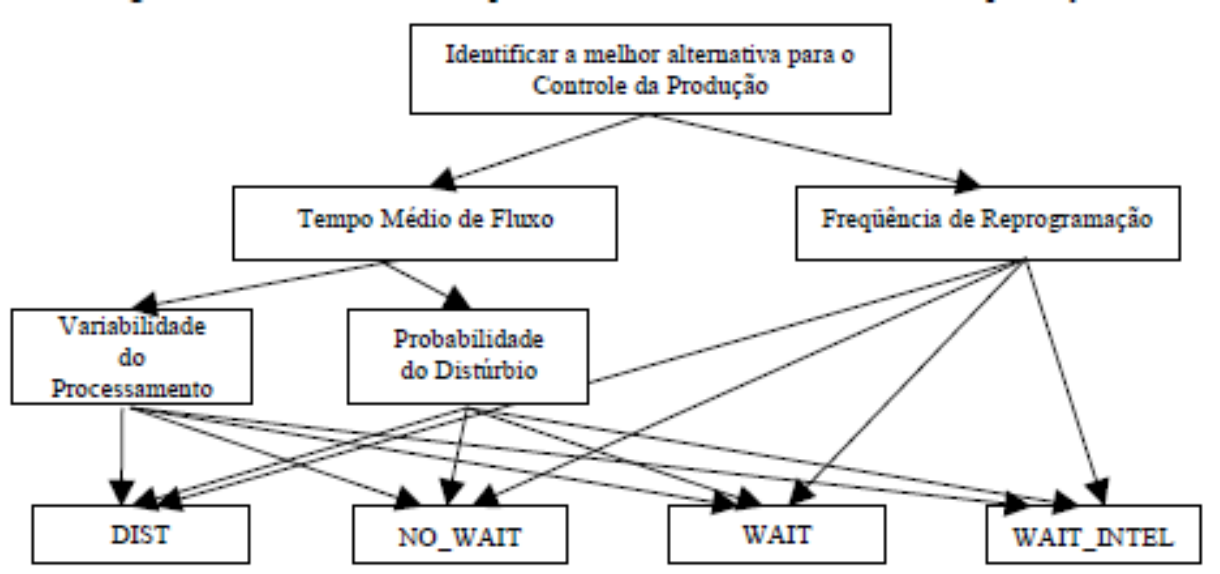


Inicialmente, a importância relativa entre os atributos deve ser estabelecida. O Tempo Médio de Fluxo é considerado a principal medida para benchmarking no controle da produção e a Freqüência de Reprogramação é considerada uma "vantagem adicional" (CAVALIERI et al., 1999). Assim, na matriz de julgamento, apresentada na Tabela 2, utilizando-se a Escala Fundamental (SAATY, 1980), considera-se o Tempo Médio de Fluxo "fortemente mais importante" que a Freqüência de Reprogramação. Os componentes do vetor de importância dos atributos, ou autovetor, apresentado na Tabela 2, podem ser obtidos com a normalização da média geométrica dos componentes da respectiva linha na matriz de julgamentos.

Tabela 2 - Julgamento e importância dos atributos para a análise do controle da produção

\begin{tabular}{|c|c|c|c|}
\hline Atributo T & T & F & Importância \\
\hline Tempo Médio de Fluxo (T) & 1 & 5 & $83,3 \%$ \\
\hline Freqüência de Reprogramação (F) & - & 1 & $16,7 \%$ \\
\hline
\end{tabular}

A dimensão dos atributos Tempo Médio de Fluxo e Freqüência de Reprogramação não é a mesma. Para que os valores de desempenho das alternativas apresentados na Tabela 1 possam ser comparados, um procedimento que pode ser utilizado para atributos inversos é a harmonização dos valores. A Tabela 3 apresenta a harmonização dos valores de desempenho das alternativas com relação ao Tempo Médio de Fluxo, assumindo-se a mesma importância para a Variabilidade do Processamento e a Probabilidade do Distúrbio.

Tabela 3 - Desempenho das alternativas de controle da produção com relação ao Tempo Médio de Fluxo

\begin{tabular}{|c|c|cc|}
\hline Alternativa & Tempo Médio de Fluxo (min) & \multicolumn{2}{|c|}{ Desempenho } \\
\hline DIST & $(139+170) / 2=154,5$ & $154,5 / 643,0=4,1618$ & $\rightarrow 4,1618 / 16,0413=25,9 \%$ \\
\hline NO_WAIT & $(145+172) / 2=158,5$ & $158,5 / 643,0=4,0568$ & $\rightarrow 4,0568 / 16,0413=25,3 \%$ \\
\hline WAIT & $(167+183) / 2=175,0$ & $175,0 / 643,0=3,6743$ & $\rightarrow 3,6743 / 16,0413=22,9 \%$ \\
\hline WAIT_INTEL & $(140+170) / 2=155,0$ & $155,0 / 643,0=4,1484$ & $\rightarrow 4,1484 / 16,0413=25,9 \%$ \\
\hline Soma & 643,0 & 16,0413 & $100 \%$ \\
\hline
\end{tabular}

A Freqüência de Reprogramação também se trata de um atributo inverso. Observa-se que a alternativa, WAIT, apresenta um valor de desempenho igual a zero. A harmonização dos dados numéricos disponíveis para este atributo implica na obtenção de um valor de desempenho igual a $100 \%$ para a alternativa WAIT e $0 \%$ para as demais.

A Tabela 4 apresenta a matriz de decisão, ou seja, uma matriz composta pelos valores de desempenho das alternativas para cada atributo. Multiplicando-se esta matriz pelo vetor de importância dos atributos, apresentado na Tabela 2, obtém-se o vetor de decisão, ou vetor de Desempenho Global, também apresentado na Tabela 4. 
Tabela 4 - Desempenho das alternativas para cada atributo e Desempenho Global

\begin{tabular}{|c|c|c|c|}
\hline Alternativa & Tempo Médio de Fluxo & $\begin{array}{c}\text { Freqüência de } \\
\text { Reprogramação }\end{array}$ & Desempenho Global \\
\hline DIST & $25,9 \%$ & $0 \%$ & $21,6 \%$ \\
\hline NO_WAIT & $25,3 \%$ & $0 \%$ & $21,1 \%$ \\
\hline WAIT & $22,9 \%$ & $100 \%$ & $35,8 \%$ \\
\hline WAIT_INTEL & $25,9 \%$ & $0 \%$ & $21,6 \%$ \\
\hline
\end{tabular}

Os resultados apresentados na Tabela 4 contradizem a conclusão de Brennan e O (2004) que "as alternativas que permitem reprogramação da produção para tratar de distúrbios resultam num melhor desempenho". Afinal, WAIT é, justamente, a alternativa que não permite reprogramação. A razão para tal contradição está nos valores utilizados como desempenho das alternativas. Na Tabela 3 observa-se que os valores do Tempo Médio de Fluxo das quatro alternativas estão na mesma ordem de grandeza: de 154,5 a 175,0 minutos. Assim, a amplitude de 20,5 minutos resultou na amplitude de 3,0 pontos percentuais. Contudo, no ambiente da manufatura, 20 minutos a mais, ou a menos, possuem uma importância maior que 3,0 pontos percentuais.

A harmonização dos valores de Freqüência de Reprogramação, conforme apresentada na Tabela 4, representa uma perda de informação. Isto se deve ao fato de existir diferenças nos valores de desempenho das alternativas com relação a este atributo.

Uma maneira de se preservar informação e de se trabalhar com dados que representem melhor as situações práticas é a utilização de julgamentos. Na próxima seção estão apresentadas matrizes de julgamentos para os desempenhos das alternativas, além da síntese dos resultados obtidos com a aplicação do método AHP.

\section{Utilização de matrizes de julgamentos}

A Tabela 5 apresenta uma matriz de julgamentos com relação ao desempenho das alternativas no atributo Tempo Médio de Fluxo. Para estes julgamentos foi utilizada a mesma escala da Tabela 2, ou seja, a Escala Fundamental (SAATY, 1980). Observa-se que julgou-se a alternativa WAIT com um desempenho fortemente pior que os das demais. Conseqüentemente, o desempenho desta alternativa, estimado conforme procedimento adotado na Tabela 2, ficou bem abaixo das demais.

Tabela 5 - Julgamentos e desempenho das alternativas com relação ao Tempo Médio de Fluxo

\begin{tabular}{|c|c|c|c|c|c|}
\hline Alternativa & DIST & NO_WAIT & WAIT & WAIT_INTEL & Desempenho \\
\hline DIST & 1 & 3 & 7 & 1 & $40,9 \%$ \\
\hline NO_WAIT & - & 1 & 7 & 1 & $23,6 \%$ \\
\hline WAIT & - & - & 1 & $1 / 7$ & $4,4 \%$ \\
\hline
\end{tabular}


Uma vantagem do método AHP com relação a outros métodos de MCDM é que, na aplicação do AHP, é possível checar a qualidade dos dados de entrada. Ou seja, no método AHP, verifica-se a coerência dos julgamentos com uma análise do autovalor, $\lambda$, da matriz de julgamentos. Caso todos os julgamentos sejam coerentes entre si, o autovalor máximo, $\lambda_{\max }$, será igual à ordem da matriz. Segundo Saaty (1980), para matrizes recíprocas positivas, $\lambda_{\max }$ pode ser estimado com o produto de um vetor composto pela soma das colunas da matriz de julgamentos pelo autovetor. Para os julgamentos apresentados na Tabela 5 tem-se:

$$
\lambda_{\max }=\left[\begin{array}{llll}
52 / 21 & 36 / 7 & 22 & 22 / 7
\end{array}\right]\left[\begin{array}{c}
40,9 \% \\
23,6 \% \\
4,4 \% \\
31,1 \%
\end{array}\right] \cong 4,17
$$

Saaty (2001) propõe um indicador da incoerência dos julgamentos, a Razão de Coerência (CR, Consistency Ratio), calculada a partir de $\lambda_{\max }$ e de constantes proporcionais à ordem da matriz de julgamentos. Para matrizes de julgamentos de 4 elementos, a CR é calculada com a equação 1 :

$$
C R=\frac{\lambda_{\max }-4}{2,67}
$$

Para os julgamentos apresentados na Tabela 5, tem-se CR $=6,36 \%$. Saaty (2001) recomenda que, para valores de CR acima de 20\%, os julgamentos sejam revistos. Contudo, Saaty (1993 apud GOMES et al., 2003) nota que a incoerência entre os julgamentos deve servir mais como um alerta do que um fato necessariamente não desejável. De acordo com Saaty (2001), a revisão dos julgamentos é um procedimento sistemático para a melhoria da tomada de decisão.

A Tabela 6 apresenta uma matriz de julgamentos do desempenho das alternativas de acordo com a Freqüência de Reprogramação. Observa-se uma pequena incoerência entre os julgamentos: julgou-se que a alternativa WAIT apresenta um desempenho absolutamente superior aos das demais alternativas (valor “9” na Escala Fundamental, SAATY, 1980); mas, também se julgou que há diferença no desempenho da alternativa WAIT_INTEL com as outras duas (DIST e NO_WAIT). Assim, o valor obtido $\lambda_{\max }$ para a matriz apresentada na Tabela 6 é igual a 4,22. Contudo, esta pequena incoerência pode ser aceita, por gerar uma razão de incoerência igual a 8,24\%. 
Tabela 6 - Julgamentos e desempenho das alternativas com relação à Freqüência de Reprogramação

\begin{tabular}{|c|c|c|c|c|c|}
\hline Alternativa & DIST & NO_WAIT & WAIT & WAIT_INTEL & Desempenho \\
\hline DIST & 1 & 1 & $1 / 9$ & $1 / 3$ & $6,2 \%$ \\
\hline NO_WAIT & - & 1 & $1 / 9$ & $1 / 3$ & $6,2 \%$ \\
\hline WAIT & - & - & 1 & 9 & $73,5 \%$ \\
\hline WAIT_INTEL & - & - & - & 1 & $14,1 \%$ \\
\hline
\end{tabular}

A Tabela 7 apresenta uma nova matriz de decisão, composta pelos novos valores de desempenho das alternativas para cada atributo. Multiplicando-se esta matriz pelo vetor de importância dos atributos, apresentado na Tabela 2, obtém-se um novo vetor de Desempenho Global. Assim, DIST passa a ser a alternativa de controle da produção com maior valor de Desempenho Global.

Tabela 7 - Novos valores de desempenho das alternativas para cada atributo e de desempenho global

\begin{tabular}{|c|c|c|c|}
\hline Alternativa & Tempo Médio de Fluxo & $\begin{array}{c}\text { Freqüência de } \\
\text { Reprogramação }\end{array}$ & Desempenho Global \\
\hline DIST & $40,9 \%$ & $6,2 \%$ & $35,1 \%$ \\
\hline NO_WAIT & $23,6 \%$ & $6,2 \%$ & $20,7 \%$ \\
\hline WAIT & $4,4 \%$ & $73,5 \%$ & $16,0 \%$ \\
\hline WAIT_INTEL & $31,1 \%$ & $14,1 \%$ & $28,2 \%$ \\
\hline
\end{tabular}

A Figura 2 apresenta a variação do Desempenho Global das alternativas em função da importância relativa do atributo Tempo Médio de Fluxo. Observa-se que a alternativa DIST apresentará o maior valor de Desempenho Global, caso ao Tempo Médio de Fluxo se atribua uma importância um pouco maior que 65\%. E isto de fato ocorre (Tabela 2). Assim, a Análise da Sensibilidade contribui para o indicativo de DIST como a melhor alternativa para o controle da produção.

Figura 2 - Variação do Desempenho Global das alternativas de controle da produção

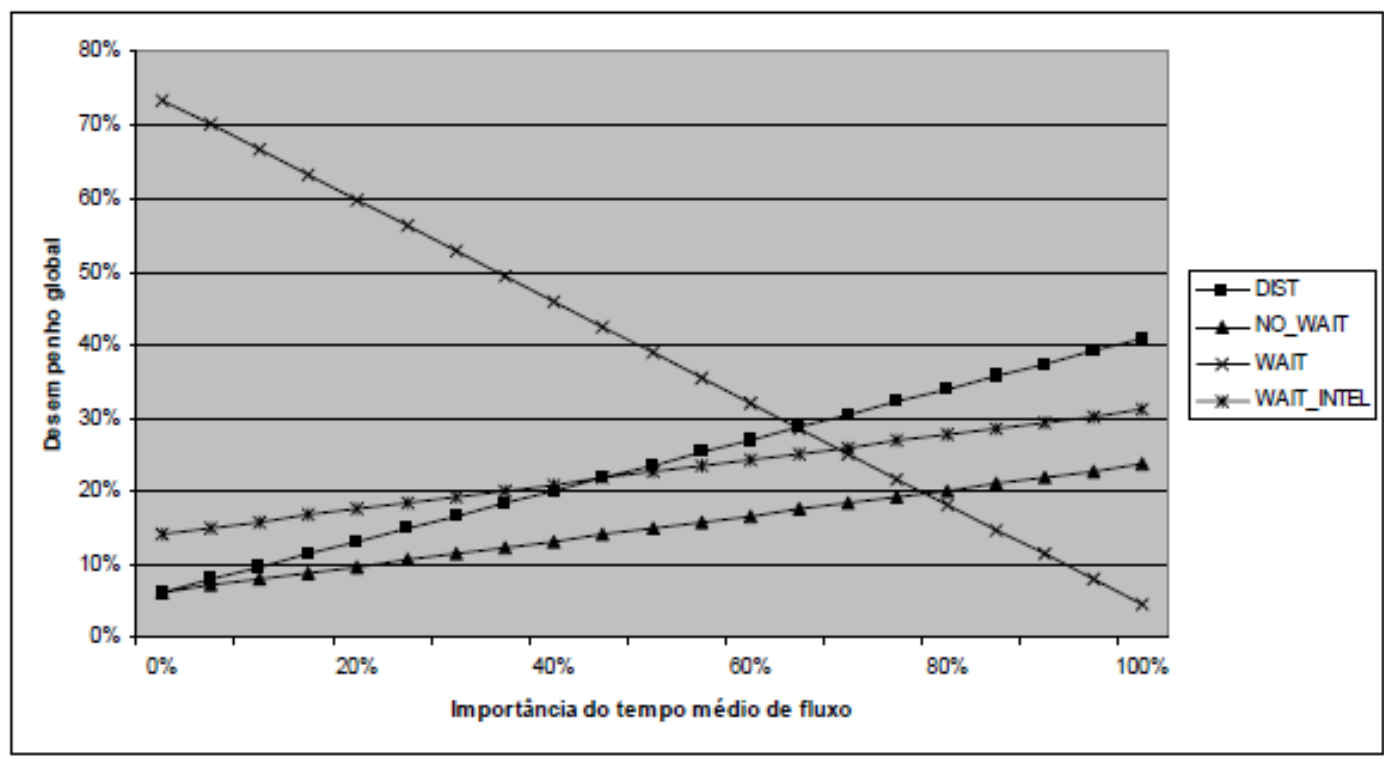


Como outros resultados da Análise da Sensibilidade, nota-se que a alternativa WAIT apresentará um maior desempenho caso à Freqüência de Reprogramação se atribua uma importância maior que 35\%. Por último observa-se que as alternativas DIST e WAIT_INTEL dominam a alternativa NO_WAIT. Na prática isto significa que, na ocorrência de algum distúrbio, vale à pena esperar um pouco ao invés de solicitar imediatamente uma nova reprogramação.

Salienta-se que os resultados apresentados nesta seção são válidos para o sistema de produção estudado por Brennan e O (2004). Além disso, estes resultados se baseiam na aplicação do método AHP com apenas dois atributos (Tempo Médio de Fluxo e Freqüência de Reprogramação).

\title{
4. Considerações Finais
}

O presente artigo apresentou a aplicação de um método de MCDM na análise do controle da produção. Dois atributos inversos foram considerados. Dados numéricos foram obtidos do trabalho de Brennan \& O (2004). Inicialmente, apresentou-se a harmonização dos dados. Os resultados obtidos não foram satisfatórios. Ou seja, não se mostraram adequados.

Matrizes de julgamentos foram então utilizadas com a aplicação do método AHP. Os resultados se mostraram adequados e mais concisos. Com a aplicação do método AHP, chegou-se à indicação de uma alternativa em função da importância relativa dos atributos.

Como propostas para novas pesquisas, surge a utilização do modelo de MCDM (atributos e alternativas) apresentado neste trabalho em estudos de casos. Outra fonte de pesquisa é a inclusão de novos atributos, como, Satisfação dos Clientes (estimado, por exemplo, com atrasos ou não atendimento de ordens de produção) ou Custos do Controle da Produção (medidos, por exemplo, a partir dos níveis de estoque).

\begin{abstract}
This article is about the multiple criteria decision making use on production control analysis. It is presented the use of judgments matrices for quantitative attributes analysis. Due to some attributes features and to the alternatives performance values, the use of a multiple criteria decision making method, with judgments matrices provides better results and a more concise analysis on the performance of production control alternatives.
\end{abstract}

Keywords: AHP, Production Control, MCDM. 


\section{Referências}

BRENNAN, R. W.; O, W. Performance analysis of a multi-agent scheduling and control system under manufacturing disturbances. Production Planning \& Control, n. 2, v. 15, 2004.

cross'

CAVALIERI, S.; BONGAERTS, L.; MACCHI, M.; TAISCH, M.; WYNS, J. A benchmark framework for manufacturing control. In. INTERNATIONAL WORKSHOP ON INTELLIGENT MANUFACTURING SYSTEMS. 2. Proceedings.

Katholieke Universiteit Leuven, 1999.

GOMES, L. F. A. M.; GONZÁLEZ A., M. C.; CARIGNANO, C. Tomada de Decisão em Cenários Complexos: introdução aos métodos discretos do apoio multicritério à decisão. São Paulo: Pioneira Thomson Learning, 2003.

SAATY, T. L. The Analytic Hierarchy Process. New York: McGraw-Hill, 1980.

SAATY, T. L. Decision Making with Dependence and Feedback: the Analytic Network Process, 2. ed. Pittsburgh: RWS Publications, 2001.

SILVER, E. A.; PYKE, D. F.; PETERSON, R. Inventory Management and Production Planning and Scheduling, 3. ed. New York: John Wiley e Sons, 1998.

SLACK, N.; CHAMBERS, S.; JOHNSTON, R. Administração da Produção, 2. ed. São Paulo: Atlas, 2002.

\section{Dados completos do autor:}

Nome completo: Valério Antonio Pamplona Salomon

Filiação institucional: Faculdade de Engenharia de Guaratinguetá (FEG)

Universidade Estadual Paulista (UNESP)

Função ou cargo ocupado: Professor Assistente Doutor

Endereço completo para correspondência (bairro, cidade, estado, país e CEP):

Departamento de Produção (Bloco 2) - Faculdade de Engenharia, UNESP

Av. Dr. Ariberto P. Cunha, 333 - CEP 12.516-410, Guaratinguetá/SP, Brasil

Telefones para contato: 12) 3123-2856/ FAX: (12) 3123-2855

e-mail: salomon@,feg.unesp.br

Recebido para publicação em: 11/11/2005

Aceito para publicação em: 19/02/2006 